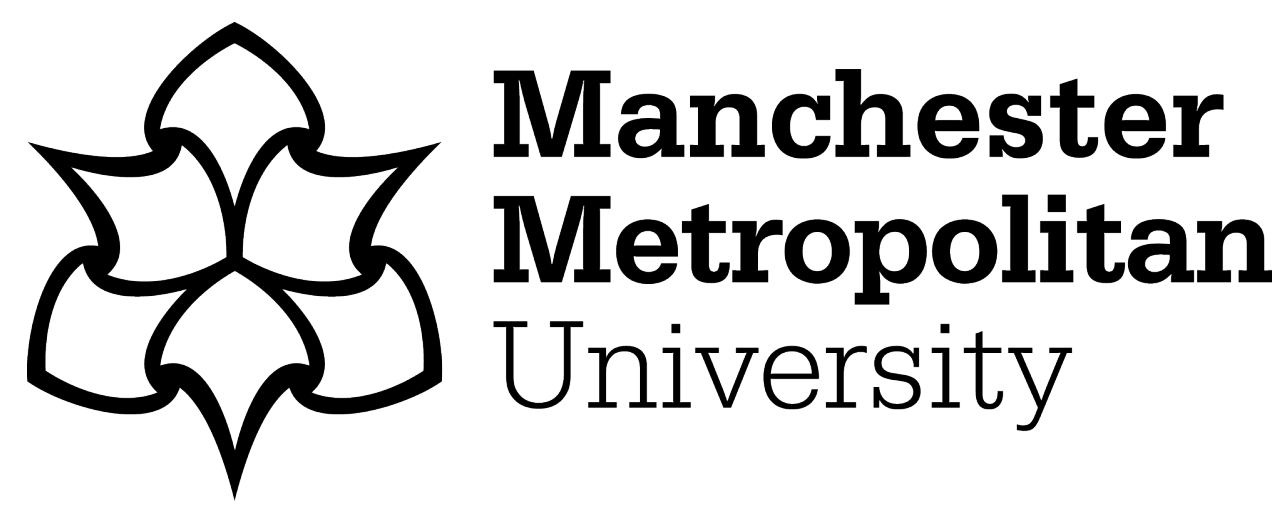

Zhao, Sumin and Flewitt, Rosie ORCID logoORCID: https://orcid.org/00000003-1986-0644 (2020) Young chinese immigrant children's language and literacy practices on social media: a translanguaging perspective. Language and Education, 34 (3). pp. 267-285. ISSN 0950-0782

Downloaded from: https://e-space.mmu.ac.uk/624150/

Version: Accepted Version

Publisher: Taylor \& Francis

DOI: https://doi.org/10.1080/09500782.2019.1656738

Please cite the published version 


\title{
Young Chinese immigrant children's language and literacy practices on social media: a translanguaging perspective
}

\author{
Sumin Zhao (a) and Rosie Flewitt (b)
}

a) Linguistics \& English Language, University of Edinburgh, Edinburgh, UK;

b) Institute of Education, University College London, London, UK

\section{ABSTRACT}

In this paper, we present a research approach that makes visible how young children in Chinese immigrant families muster their multilingual, multimodal and multisemiotic repertoires as they interact with distant family and friends on social media. The approach brings multimodal social semiotics into conversation with translanguaging to problematize the notion of languages as bounded systems, and to illustrate how emergent multilingual learners deploy their knowledge of the features of different language scripts and modalities to maximise their communicative capacity. We focus on the emergent translanguaging practices of Chinese immigrant children when using WeChat - a popular Chinese social media that is widely used by young families in their everyday language and literacy practices. Reporting on a study of nine immigrant families in southeast London, we home in on one boy aged eight years and his younger brother aged six years, with mixed Chinese (mother) and Portuguese (father) immigrant heritage. Through fine-grained multimodal analysis of online exchanges between the older brother and contacts in their mother's WeChat network, we illustrate the multimodal, translinguistic and polyadic nature of his language use in practice and reflect briefly on the disjuncture between his home uses of multiple languages and his schooling. We also consider how the younger brother is socialised into translanguaging practices by observing and occasionally participating in his older sibling's online chat. The findings address a gap in research knowledge by illustrating how social media can enrich opportunities for young children's emergent translanguaging practices and heritage language learning.

\section{Introduction}

Reflecting a global rise in migration, almost 1 million children in England who currently attend state-funded primary schools live in homes where languages other than English are spoken. This trend has been growing steadily, rising from $10 \%$ of primary-aged children in 2003 to $16 \%$ in 2010 and over $21 \%$ in 2018 (DfE, 2003, 2010, 2018). One of the many challenges facing these young children is the disjuncture between their multilingual out-ofschool and often monolingual in-school language and literacy practices. In English speaking countries, particularly where schools adhere to 'English-only' policies (Axelrod and Cole 2018), longitudinal analyses suggest that children who speak a language other than English at home often experience a decline in heritage language use during early childhood, and heritage language loss after starting formal schooling (Verdon, McLeod and Winsler 2014), which impacts negatively on familial relations and individuals' sense of identity (Wong Fillmore 2000). Multiple factors contribute to language loss including the lack of language input (Pearson 2007), the language choice of siblings (Pease-Alvarez 1993), pressure from preparation for schooling in English (Jordaan 2008), and low social status of the heritage language (Dixon, $\mathrm{Wu}$, and Daraghmeh 2012). We argue that there is a pressing need for research into young multilingual children's meaning making across languages and modalities, 
which has profound implications for their identity, for pedagogical practice and for the mitigation of heritage language loss.

While studies of young children's heritage language maintenance practices have focused largely on home factors such as familial attitudes (Park and Sarkar 2007), parental language input (Scheele, Leseman, and Mayo 2010) and support (Mattheoudakis, Chatzidaki, and Maligkoudi 2017), an emerging body of literature is drawing attention to the new opportunities and affordances for multilingual exchanges offered by digital technologies. Immigrant parents are reported to be generally positive about the role new technologies can play in facilitating heritage language and culture practices, and often actively participate in selecting digital resources to support their children's language learning ( $\mathrm{Hu}$, Torr, and Whiteman 2014; Szecsi and Szilagyi 2012). This learning can occur both around and through technology use. Kenner et al. (2008) and Akhter (2016), for instance, have shown how the co-use of computers facilitates linguistic and cultural exchange between grandparents and grandchildren, and supports young children's learning about heritage language and literacy. In this article, we explore the roles that social media can play in fostering heritage language maintenance and cultural practices by connecting young immigrant children with their distant heritage communities, which in turn offers rich opportunities for the development of communicative repertoires that extend across languages and modalities. We present findings from a study of nine immigrant families of Chinese background with young children aged between three to eight years, who were living in southeast London. We found that these families frequently used WeChat, a popular Chinese-language social media app, to connect with their Chinese-speaking families and friends. Focussing on one case study of a family with two boys aged eight and six years living with their Chinese immigrant mother and Portuguese immigrant father, we interrogate the WeChat exchanges between the older brother, Dawei, and diverse contacts in his mother's social network. We also observe how the younger brother, Xiaowei, engaged in these exchanges through 'intent participation' (Rogoff et al. 2003), that is, by observing intently and occasionally participating more actively in his brother's use of social media.

To understand the linguistic and modal complexity of the boys' online practices, we adopted the dual lenses of translanguaging ( $\mathrm{Li} 2018$ ) and a social semiotic approach to multimodality (Halliday 1978; Kress and Van Leeuwen 2001). We suggest that translanguaging offers a practical theory of language that enables the different languages used by multilingual speakers to be conceptualised as part of an integrated communication system. This perspective is complemented by multimodality, which sees young children as meaningmaking agents who deploy multiple semiotic resources (linguistic, visual, aural, embodied) in their meaning-making practices. Our analysis highlights the creative and inventive ways in which young emergent bilingual children use the multimodal and multilingual resources available in WeChat as they become socialised in social media practices and in their family heritage cultures. We argue that social media can provide impetus and learning opportunities for young children to build their heritage language and literacy as part of an integrated repertoire of meaning making (Canagarajah 2011; Axelrod and Cole 2018) through transnational and translinguistic encounters (Hawkins 2018).

\section{Young children's translinguistic and transnational communication on social media}

The ubiquitous presence of social and mobile media in contemporary life has led to new communication practices that are often both multilingual (Androutsopoulos 2015; Dovchin 2017), and multimodal (Zhao and Zappavigna, 2018). Social media have become 'mediated 
sites' (Reershemius 2017, 45) for language learning, offering new opportunities for learner agency and motivation, with calls to redesign language learning for the digital age (KukulskaHulme 2012). Research into the role that social media play in heritage language practices immigrant or autonomous - and maintenance has focused principally on adolescents and adults (Jones and Uribe-Jongbloed Gruffydd 2012; Stewart 2014; Velazquez 2017), and very little is known about younger children's social media communication practices. This is understandable given that social media platforms set a minimum age limit (typically 13 years) for their users.

Nevertheless, research has shown that young children aged under eight participate actively online in social media practices (Holloway, Green, and Livingstone 2013). An emerging body of qualitative research has begun to show how young children are socialised into social media literacy practices as part of everyday family life (Marsh et al. 2017), and how these practices offer out-of-school learning opportunities that are often mediated by parents (Zaman et al. 2016). Other studies have highlighted the role that video-based social media (e.g. Skype) can play in supporting distance intergenerational literacy activities such as pretend and narrative play (Kelly 2013). Literature on young children's social media and digital technologies in general, however, tends to focus on monolingual children and there is very little work on multilingual children's language learning opportunities in social media.

Our study speaks to this gap in knowledge by exploring emergent bilingual children's translinguistic and multimodal practices on the Chinese language social media platform WeChat, known as Weixin in Chinese. Launched in January 2011, WeChat is currently the fifth most popular social media service globally, with over one billion active users (Statistica 2018). For the highly mobile - both internally and internationally - Chinese population, WeChat is the main channel for distance intergenerational communication (Yu, Huang, and Liu 2017; Zhou and Gui 2017). In a study of Chinese young adults' interactions with their geographically distant parents, Yu, Huang, and Liu (2017) identified three different styles of WeChat interaction: conversational (video and voice calls); connected (short, frequent and spontaneous one-to-one chats for both expressive and instrumental goals); and panoptic (family group chats, and performative interaction where the main purpose is to project a sense of positivity to alleviate their parents' concerns). WeChat also provides a site for transnational literacy learning, as suggested by Wang's (2017) recent study of Chinese university students in the UK.

\section{Bringing multimodality to translanguaging theory}

To capture the complexity of how global communications systems impact on young multilingual children's translinguistic and multimodal interactions using social media, we bring close-textured multimodal social semiotic analysis (Halliday 1978; Kress 2010) to translanguaging theory. We turned to Garcia and Li (2014) and Li's (2018) conceptualisation of translanguaging to help us understand how emergent bilingual children draw on their developing knowledge of multiple language and literacy conventions when interacting with distant friends and family on social media. Rather than treating different languages as separate, bounded systems that bilingual children move between as they switch codes, a translanguaging perspective views bilinguals as drawing fluidly across the repertoire of multiple languages with which they are familiar. Translanguaging therefore conceptualizes language as an activity - as something language users do, rather than as a structure that they draw on. Recent research has begun to report on emergent bilinguals' translanguaging practices, including in early childhood settings (Gort and Sembiante 2015; Pontier and Gort 
2016; Schwartz and Asli 2014) and in primary education (Bauer, Presiado, and Colomer 2017). These studies all suggest that young children deploy all their language resources in rich linguistic repertoires, producing speech and writing that contain elements of multiple language systems. As children become more competent in multiple languages, so they distinguish between discrete language conventions, and tend to use languages in more standard ways (Gort 2006).

Translanguaging theory mirrors the emergence of similar terms to describe linguistic practices where languages are used flexibly in communicative repertoires, such as code-meshing (Canagarajah 2011), flexible bilingualism (Creese and Blackledge 2015) and translinguistic practice (Canagarajah 2015). In line with the New London Group's (The New London Group 1996) account of multiliteracies, these approaches all conceptualise language as a complex social resource, and place the speaker rather than the language at the centre of analysis. Pennycook (2017) further argues that language is only one semiotic resource amongst many, and that translanguaging theory enables researchers to attend to 'not only the borders between languages but also to the borders between semiotic modes' (p270).

This theoretical framing therefore resonates with work in the field of multimodality, which has illustrated how young children's 'semiotic resourcefulness' (Mavers 2007, 155) is integral to their multimodal meaning making, from text making (Mavers 2011) to storytelling (Harrop-Allin 2014), and from digital art creation (Sakr and Kucirkova 2017) to physical play (Cowan 2018). In the current era of digital communication, young children's digitally mediated interactions are shaped by the affordances and constraints of the multimodal resources available across the diverse social mediascapes that they inhabit, and by the conventions inherent in these comparatively new contexts of use. We found that bringing a social semiotic multimodal perspective to translanguaging theory enabled us to develop an approach that offered rich and fine-grained insights into young emergent bilingual children's contemporary language and literacy practices on social media.

\section{Study design and process}

The case analysed in this paper originates from a small-scale, qualitative study of nine Chinese immigrant families, conducted in southeast London in 2016 (for detail of wider project findings, see Zhao 2019). The main research question addressed in this paper is:

- How do young children in Chinese immigrant families in England deploy the range of linguistic and other semiotic resources available in social media to interact with distant friends and family?

Opportunistic sampling was used, based on our own contacts (three families), which led us to a Chinese Saturday school in southeast London and contact with six more families. The nine families, with 12 children aged under eight, were diverse in terms of parental education and profession, immigration history, socio-economic backgrounds, and parental proficiency in English and heritage languages. Data collection began with face-to-face semi-structured interviews with the children's parents. Through these interviews, we identified three families that reported substantial use of WeChat by the children for learning about their heritage language and culture. We subsequently observed these three families using WeChat at home, and gained further insights into each family's WeChat practices through unstructured interviews with parents and children. 


\section{Participants and setting}

In this paper, we focus on one case study family's use of social media at home and report on patterns of interaction that we observed across the participating families. The mother, a Mandarin Chinese speaker in her $40 \mathrm{~s}$, had moved to London ten years previously to pursue her MBA study. At the time of data collection, she combined managing an online business with home and parenting responsibilities. The father, a European Portuguese speaker in his $50 \mathrm{~s}$, had lived in the UK for 15 years and ran a small independent business. Their only relative in the UK was a distant cousin of the mother. The brothers - Dawei (aged eight) and Xiaowei (aged six) — were both born in London and attended a local state primary school where the mandated language of instruction was English. The family used English to communicate with each other, but the mother spoke Chinese with the brothers from time to time. The brothers did not speak Portuguese, as their father had always spoken in English with them and did not consider it necessary for them to learn his heritage language.

The two brothers had different linguistic histories. The older brother Dawei learnt Chinese as his first language, because the mother 'was determined' that 'he should treat Chinese as his mother tongue from the very beginning'. Nevertheless, the mother reported that Dawei had experienced language loss since entering early education, when English became his main language. To help him continue to learn his mother's heritage language, at age five, he started attending a newly established community-run Saturday Chinese school, which became his main source of Chinese language learning. For a short period, Dawei's mother was a volunteer teacher at this school and taught Dawei, which she believed impacted positively on his Chinese language learning. The younger brother Xiaowei, however, learnt English as his first language from birth. Xiaowei attended the same Chinese school, but his Chinese, in their mother's view 'can't really compare with Dawei's'. Beyond the home and Saturday Chinese school, the brothers used little Chinese in any other social contexts in England.

The mother had been using WeChat since 2014 to connect with her family and friends in China and Chinese-speaking friends in the UK. When his mother used WeChat on the iPad, Dawei would often closely observe her, and noticed that she used Pinyin to type Chinese. Pinyin is the Romanization of Chinese based on a phonological coding of Mandarin Chinese. It marks the vowel, consonant(s) and diacritic tone of a character. Pinyin input allows a user to type Chinese characters by entering the Pinyin (without the diacritic tone) of a character, resulting in a list of possible characters with the same vowel and consonant(s) combination, or homophones. As Dawei was learning Pinyin at the Chinese school, he would often read out what his mother was typing, even if he did not know the corresponding character. Once he had discovered his mother's use of Pinyin, Dawei started to type Chinese characters on the iPad, asking his mother for help when he was unsure which character to choose. This allowed him to participate in his mother's WeChat conversations, which he encouraged Xiaowei to join in, and which Xiaowei observed intently.

During the project, three home visits, each lasting three and half to four hours, were made to Dawei's family with a follow-up visit one year later for an update on the family's communication practices. Informal interviews were conducted with the mother and children, along with video-recorded observations of WeChat calls between the brothers and their grandparents in China, and field notes captured wider information (see Zhao 2019 for a full report on interview data). Additionally, the mother sent us screenshots, which captured exchanges between Dawei (and Xiaowei) and various individuals or groups of 
people in the mother's extended social network in China and the UK, during a three-month period (April-June) in 2016. These included: 1) video clips made for the maternal grandparents of the brothers engaging in Chinese language and literacy practices (see Zhao 2019); and 2) screenshots of WeChat interactions between Dawei (often with Xiaowei) and distant friends and family.

Our data collection was constrained by the small-scale nature of the project, wherein we only observed WeChat conversations that occurred spontaneously and sporadically during the home visits. Many of the screenshots sent to us by the mother involved short exchanges between the mother and her contacts with only one or two moves (e.g. greetings) made by Dawei. In this paper, we focus on four extended episodes where Dawei instigated the online chat (see Appendix A for overview of these episodes). We were not present when these exchanges occurred, but interviewed Dawei and his mother about them. We acknowledge the limits of our methodology (see Zhao 2019) and suggest that a long-term ethnographic project with deep fieldwork would produce a more comprehensive dataset.

\section{Data analysis procedures}

The analysis of multimodal data such as video recordings often requires the development of sophisticated multi-tier transcription schemes (Bezemer and Mavers 2011). However, for the social media chats featured here, we developed a two-column coding scheme, with the screenshots on the left and the coding of conversational structure, language features and (by Zhao) on the right (see Figure 2). We followed a Hallidayan analytical framework (1978) to code the conversational structure, as developed by Eggins and Slade (2005), 1 and to code the interpersonal dimension, which is how social relations are enacted and negotiated in meaning making. The interpersonal features that dominate the WeChat data include linguistic lexis such as vocatives, slang and idioms, visual elements such as emojis and stickers as well as greeting and repair moves (see Figure 2). We suggest that there are two possible factors at play here: firstly, the phatic nature of social media communication (Miller 2008; Zappavigna 2012), and secondly, the emergent nature of Dawei's literacy skills in Chinese which may have constrained the ideational dimension (or topic areas) of his interaction. Our analysis is contextualised with information from interviews with the mother, field notes from home visits, follow-up conversations with the mother and Dawei, and other screenshots captured by the mother.

\section{Findings}

\section{Multimodality and translanguaging in WeChat}

The WeChat episodes were conducted in written Chinese, written English, spoken Chinese (Mandarin), and a range of image-based semiotic resources. Dawei used Pinyin to type Chinese characters and solicited occasional help from his mother to choose the right character from a list of homophonic characters. In two of the selected episodes ( $1 \& 3)$, both he and Xiaowei audio-recorded and sent short greetings in Mandarin. In addition to verbal modes, Dawei selected stylised semiotic resources available in WeChat, including visuals (emoji, kaomoji,2 and selfies), stickers (a combination of written language and image/moving image), and 'virtual' artefacts (digital red envelope3). The diversity of Dawei's semiotic choices is particularly striking when compared with the more limited semiotic range used by his (adult) interlocutors (see Table 1).

In Table 1, we see that adults used mostly written Chinese or English, whereas Dawei drew on a wide range of semiotic resources available in WeChat, often to express his feelings, and this practice was sometimes echoed in adults' responsive uses of stickers and 
emojis. Dawei frequently used stickers, which he considered 'haowan' (fun). Stickers (Lim 2015) are a type of virtual communicative gesture and are either visual (e.g. images or animated images) or combine language and visuals through 'mode-meshing' (James 2016).

\begin{tabular}{|c|c|c|c|c|c|}
\hline \multirow[t]{2}{*}{ Episodes } & \multicolumn{5}{|c|}{ Uses of modes by participants } \\
\hline & \multicolumn{3}{|c|}{ Modes } & \multirow{2}{*}{$\begin{array}{c}\text { Dawei } \\
\text { (Xiaowei) } \\
\frac{\sqrt{ }}{}\end{array}$} & \multirow{2}{*}{$\begin{array}{c}\text { Adults(s) } \\
\sqrt{ }\end{array}$} \\
\hline \multirow[t]{5}{*}{1 Do you remember me } & \multirow[t]{2}{*}{ Language } & Written & \multirow{2}{*}{ Chinese } & & \\
\hline & & Spoken & & $\sqrt{ }$ & \\
\hline & \multirow[t]{2}{*}{ Visual } & \multicolumn{2}{|l|}{ Stickers } & $\sqrt{ }$ & \\
\hline & & \multicolumn{2}{|l|}{ Emojis } & $\sqrt{ }$ & \\
\hline & Multimodal & \multicolumn{2}{|c|}{ Digital Artefacts } & $\sqrt{ }$ & $\sqrt{ }$ \\
\hline \multirow[t]{5}{*}{2 I miss you } & \multirow{2}{*}{ Language } & \multirow{2}{*}{ Written } & Chinese & $\sqrt{ }$ & \\
\hline & & & English & & $\sqrt{ }$ \\
\hline & \multirow[t]{2}{*}{ Visual } & \multicolumn{2}{|l|}{ Stickers } & $\sqrt{ }$ & \\
\hline & & \multicolumn{2}{|l|}{ Emojis } & $\sqrt{ }$ & $\sqrt{ }$ \\
\hline & Multimodal & \multicolumn{2}{|l|}{ Stickers } & $\sqrt{ }$ & \\
\hline \multirow[t]{7}{*}{3 Reunion dinner } & \multirow[t]{2}{*}{ Language } & Written & \multirow{2}{*}{ Chinese } & $\sqrt{ }$ & $\sqrt{ }$ \\
\hline & & Spoken & & $\sqrt{ }$ & \\
\hline & \multirow[t]{3}{*}{ Visual } & \multicolumn{2}{|l|}{ Stickers } & $\sqrt{ }$ & $\sqrt{ }$ \\
\hline & & \multicolumn{2}{|l|}{ Emojis } & $\sqrt{ }$ & \\
\hline & & \multicolumn{2}{|l|}{ Selfies } & $\sqrt{ }$ & \\
\hline & \multirow[t]{2}{*}{ Multimodal } & \multicolumn{2}{|l|}{ Stickers } & $\sqrt{ }$ & $\sqrt{ }$ \\
\hline & & \multicolumn{2}{|c|}{ Digital Artefacts } & & \\
\hline \multirow[t]{4}{*}{4 The other David } & \multirow[t]{2}{*}{ Language } & \multirow{2}{*}{ Written } & Chinese & $\sqrt{ }$ & \\
\hline & & & English & $\sqrt{ }$ & $\sqrt{ }$ \\
\hline & \multirow[t]{2}{*}{ Visual } & \multicolumn{2}{|l|}{ Stickers } & $\sqrt{ }$ & \\
\hline & & \multicolumn{2}{|l|}{ Kaomojis } & $\sqrt{ }$ & \\
\hline
\end{tabular}

Table 1. Use of semiotic resources across the WeChat episodes.

Detailed analysis of Dawei's conversational moves suggests he selected stickers sensitively according to the context of a specific exchange, primarily to realise and/or emphasise the interpersonal metafunction. For example, in Figure 1 - which occurred at the beginning of the Reunion Dinner exchange (Episode 3) - we see how Dawei used stickers during an exchange with two of his mother's high school classmates, a female (CF1) and a male (CM1).

This episode began with the female adult introducing herself to Dawei as “a'yi” ( 阿 姨 ， auntie) the term for both non-kinship female adults and sisters of the mother. The male adult, however, referred to himself as “jiujiu” (舅舅, maternal uncle), followed by a (handson-mouth) laughing emoji. This utterance was intended as a joke, with its humour hinging on the elaborated Chinese kinship system and terminology (see Chen 2010). Chinese speaking children address non-familial male adults as 'Shushu' (叔叔 ), which is also the term for younger brothers of the father. The interlocutor, however, introduced himself as 'Jiujiu', a term used to address younger brothers of a mother, thereby jokingly implying that 
he was 'related' to Dawei through the mother. This use of jiujiu created a 'conversational bond' (Knight 2013), shortening the social distance between the two. Dawei acknowledged this bond by replying with "haha" (哈哈), followed by a sticker of a dog rolling on the floor laughing. The function of this sticker is similar to laughter in casual conversations, signalling that Dawei is 'laughing with' (Knight 2013) his interlocutor. Dawei returned to this conversational bond in later moves (see Figure 4).

In the next move, Dawei sent two audio clips to CF1 and CM1 in spoken Chinese ('Shushu Aiyai'), but these were not immediately acknowledged by the adults. He followed up with a sticker, with the question “Youren-ma?"(有人吗? Is anyone there?) and a dog emoji with a downward-turned mouth. This acted as an appending move, and subtly added a saddened affective dimension to the exchange, in contrast with the laughing dog in the previous move. Across his exchanges on WeChat, Dawei used stickers featuring both Chinese and English. We view these conversational uses of stickers as 'emergent' translanguaging practices that mesh different linguistic, cultural and identity codes (Li 2018). We consider these translanguaging practices as emergent because as a heritage language learner, Dawei was still developing the linguistic expertise needed to blend English and Chinese at structural or rhetorical levels. The visual resources available in WeChat therefore not only served to signal the light-hearted nature of the interpersonal metafunction, but were also an enabling feature for Dawei to maintain the conversation while constructing his own identity within the exchange.

In addition to using stickers to enact the interpersonal dimension of meaning making, such as creating social bonds and expressing emotions, Dawei occasionally used stickers and emojis to facilitate the ideational dimension (or topics) of communication. This is illustrated by the Reunion Dinner extract (Figure 2), which is taken from an hour-long group chat between Dawei and his mother's high school friends in China (see Figure 3 for more detail). 


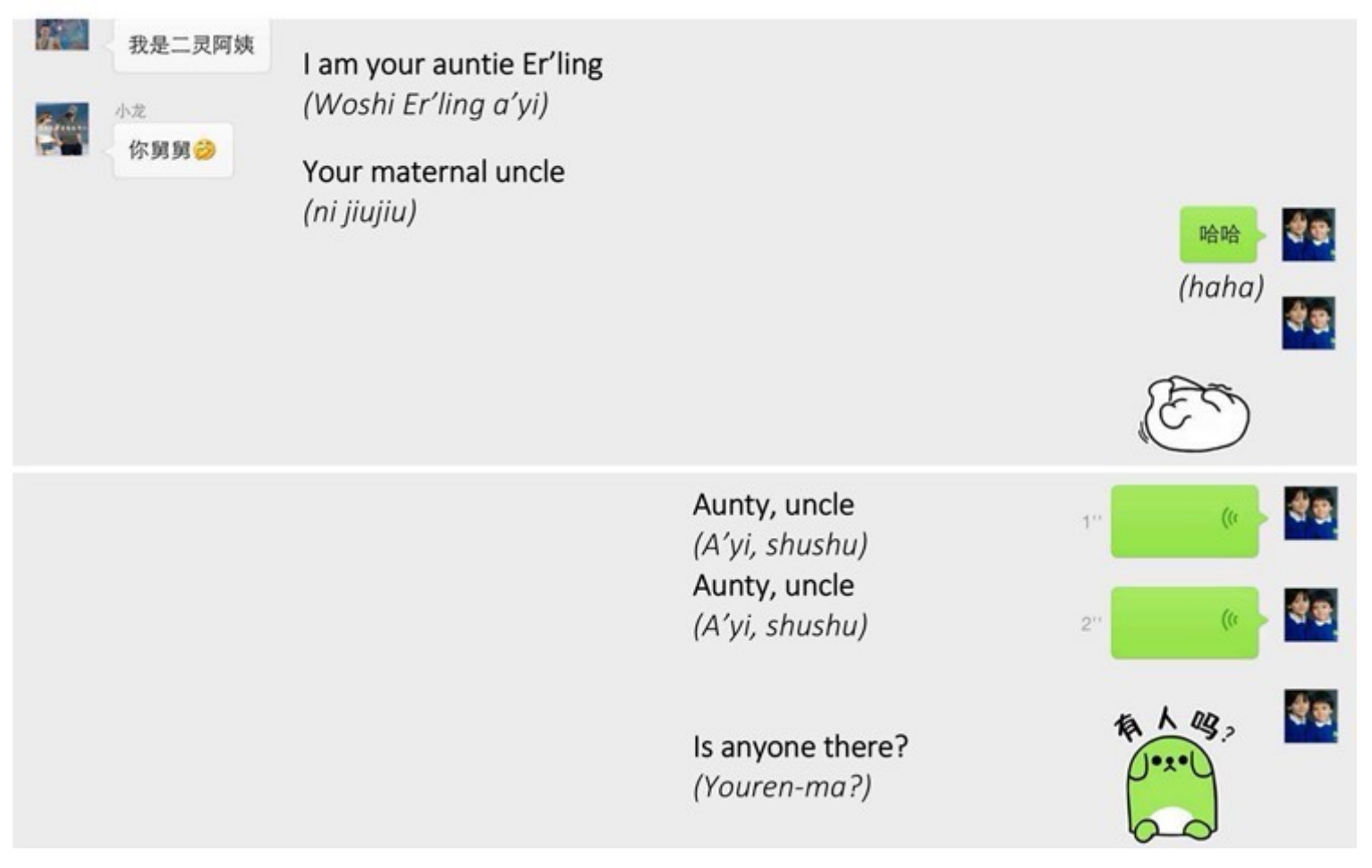

Figure 1. Dawei's conversational use of stickers.

This exchange started with Dawei asking CM2 (L42) to "wan youxi" ( 玩 游 戏, play a game), followed by a series of exchanges using rock-paper-scissors emojis. The game closed with Dawei declaring victory (L51) "Wo yinle” (我赢了, I have won) and the adult conceding defeat (L52) "Shu-le" (输了, Lost). The exchange was briefly 'interrupted' by two different adults (CM3, CF2) greeting Dawei (L43-44). This extract exemplifies Dawei's creativity and playfulness in utilising WeChat's multimodal resources. This inventiveness was critical as it allowed Dawei, whose Chinese literacy skills were still emergent, to generate new conversational contexts and move the topics beyond simple greetings and affective exchanges. The cooperative responses from CM2 provided further impetus for Dawei to use Chinese. During interview, we learnt that Dawei was frequently praised by adults when he used Chinese ("people would say "Woo, Dawei, you speak such good Chinese."'), which motivated him to continue with heritage language learning in an otherwise English dominant environment. His younger brother, in contrast, had seldom received similar encouragement ('...but Xiaowei never received such praise'). Deploying a range of semiotic resources also enabled Dawei to manage complex conversational moves in the polyadic interactional environment of $\mathrm{WeChat}$, a point of to which we now turn. 


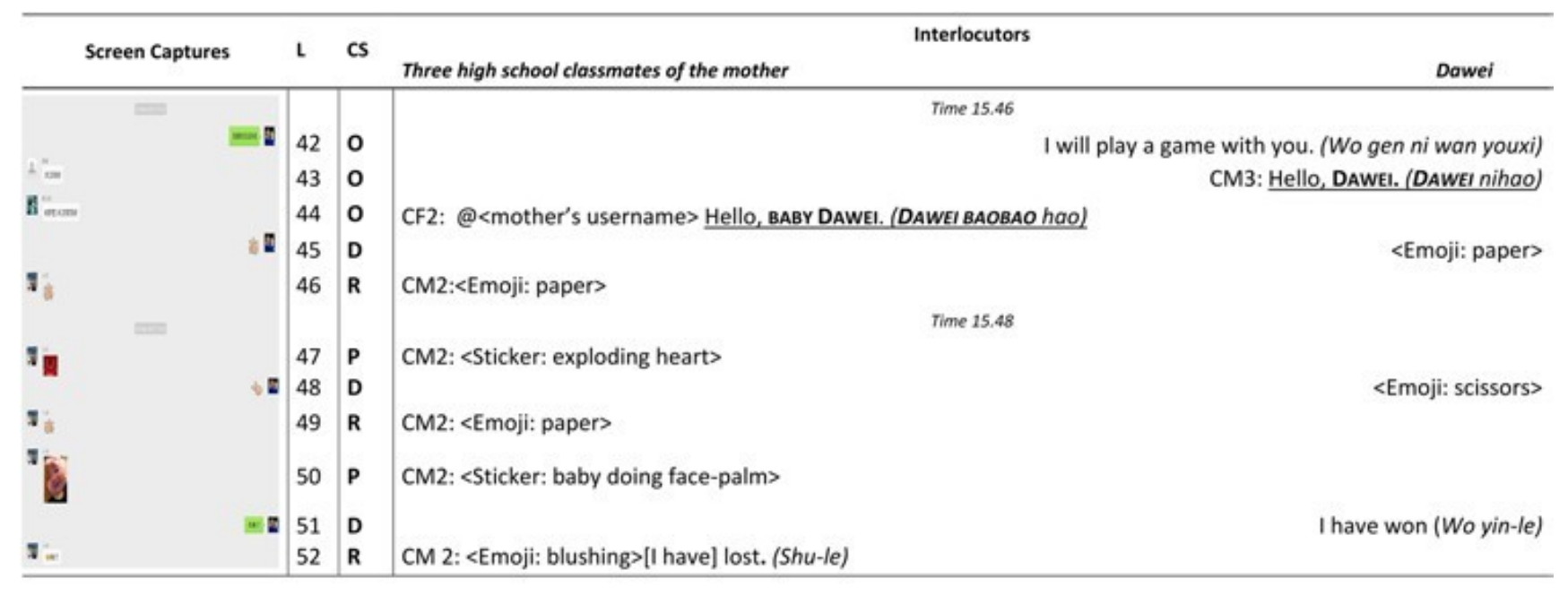

Notations:

L: Lines

CS: Conversation Structure

Involvement Lexis: VOCATIVE, IDIOMS, SLANG

O: Opening Move

R: Reacting Move

D: Developing Move

P: Prolong Move

A: Appending move

s: supporting

c: confronting

Interrupting and un-responded moves: underlined

Figure 2. Emojis and multimodal play. 


\section{The polyadic nature of WeChat interactions}

WeChat interaction is often polyadic in nature, and this feature is embedded in the platform's design (e.g. group chat function), in line with the functionality of other social media platforms. Polyadicity enables conversations between multiple partners and supports interaction across diverse conversational sites, as occurred in the Reunion Dinner episode.

Figure 3 models how three different conversations were held simultaneously. In China, the mother's school friends were enjoying their annual reunion dinner, and were interacting with each other both face-to-face and on WeChat in their high school chat group. Through the WeChat group chat, they were also chatting with former classmates in different geographic locations in China and overseas (including Dawei's mother in England).

Meanwhile, in London, Dawei, Xiaowei and their mother were visiting a Chinese friend at her home in southeast London. The mother was talking with her friend in Chinese, whilst attending to the high school group chat from time to time. At one point, Dawei took over the WeChat conversation from his mother and started an extended exchange with a group of five of his mother's friends, none of whom he had previously met. This translocal (Yu, Huang, and Liu 2017) and transnational (Wang, 2017) WeChat episode lasted over an hour and only ended when the mother and the two brothers left the friend's house to go home. Exchanges such as these extended Dawei and Xiaowei's Chinese-speaking social world and provided them with opportunities to engage in contextualised, transnational heritage language and literacy practices beyond their home and Saturday Chinese school environment.

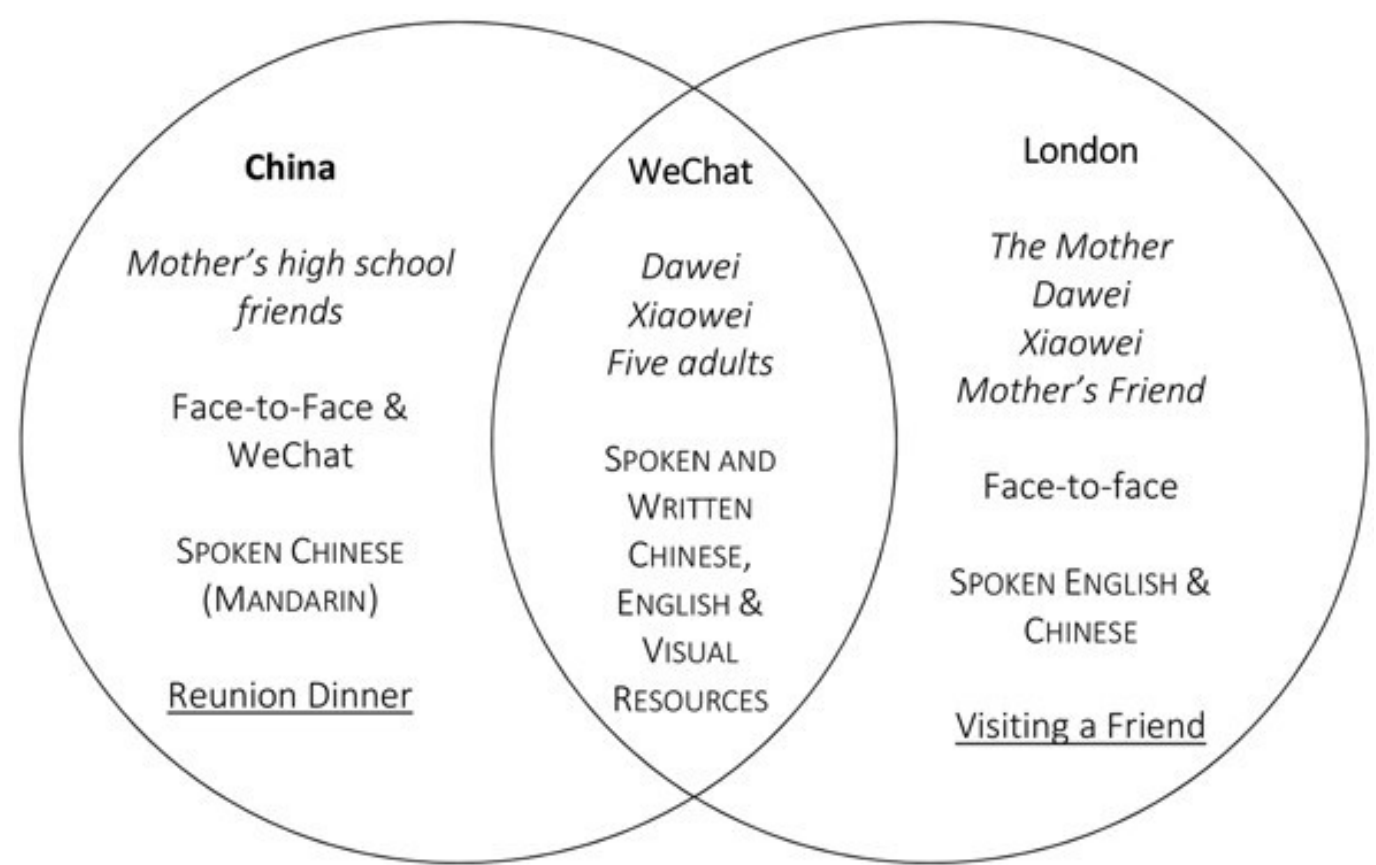

Figure 3. Polyadicity \& WeChat interaction: The Reunion Dinner.

Not only did the interactive, multimedia and multimodal affordances of WeChat lead to complex exchanges across diverse modes and languages, but the polyadic nature of WeChat interaction also resulted in the exchanges often being fragmented and frequently interrupted, with conversational topics constantly shifting and interlocutors dropping in and out of the exchange, as illustrated in Figure 4. 
This exchange began with Xiaowei introducing himself (L22), encouraged by Dawei “Nihao wo shi Xiaowei” (你好我是小卫, Hello, I am Xiaowei), CM2 replying with a kiss Emoji (L23) and CF1 praising both brothers for their Chinese (L24). Xiaowei followed up with three more moves, using written Chinese (L25), a sticker (L26) and Chinese characters as laughter tokens (L27), before Dawei returned to the conversation (L28). Dawei's first move (L28) was to re-establish his identity by informing the adults that the previous laughter token (L27) 'ha-ha' ( 哈 哈 ) had been typed by Xiaowei, to which CM2 replied (L29) in written Chinese 'Zhe hai chicu' (这还吃醋 4 Are you being jealous?). Here, Dawei may not have understood the idiomatic expression chicu, as he followed up with a second move (L30) to establish his identity as Dawei. He then attempted to re-start the exchange with a greeting, expressed through a sticker of a footballer saying "Hi" (L32). CM2 returned his greeting (L33) with "Dawei nihao" (大卫你好, Hello Dawei), to which Dawei responded (L35) “Jiujiu hao" ( 舅舅 好 , Hello, maternal uncle), referring back to the earlier conversational bond (presented in Figure 1).

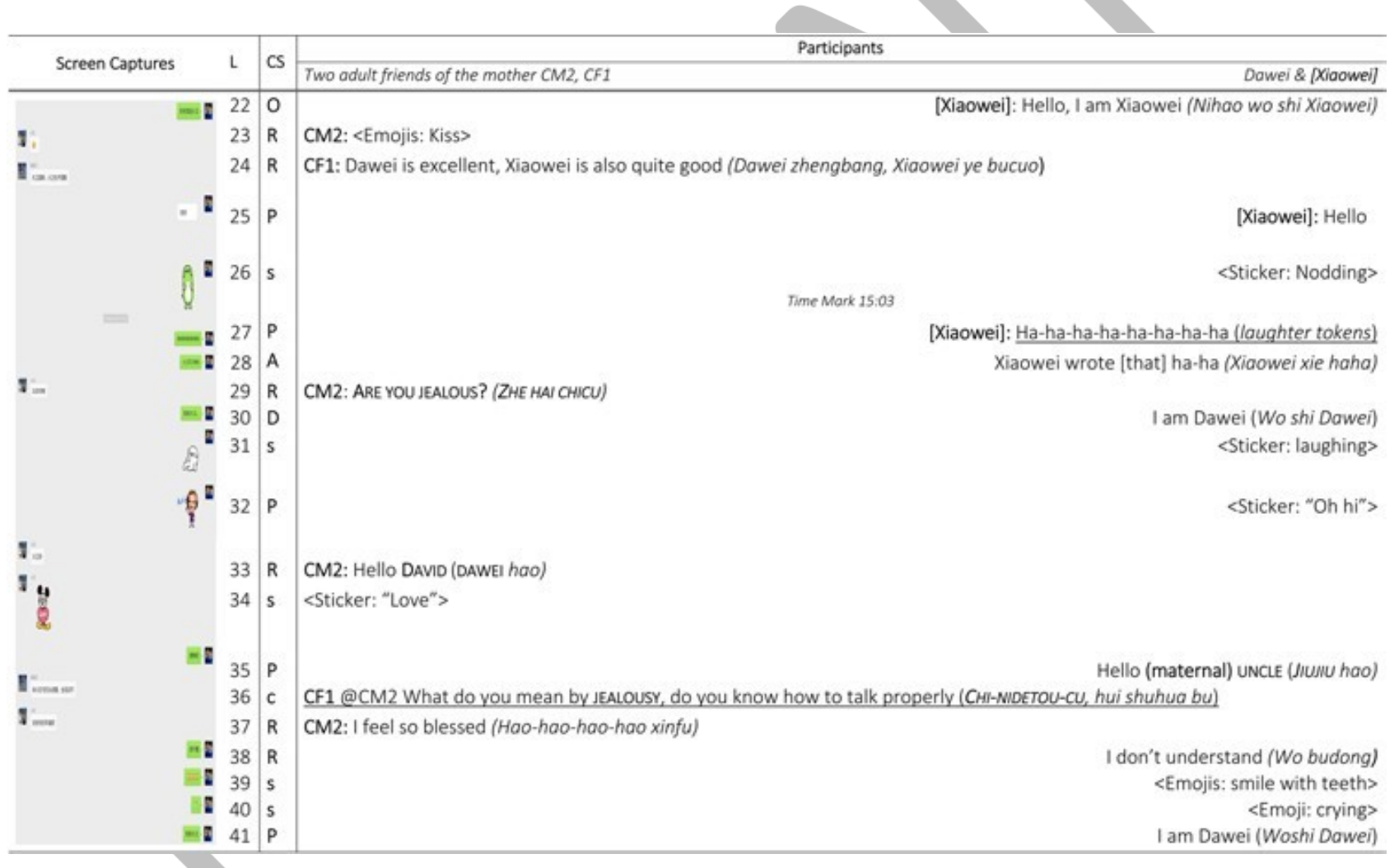

Figure 4. Managing multiple interactional partners.

At this point (L36), CF1 interjected to suggest it was inappropriate for CM2 to accuse Dawei of being jealous. The @username signals that this message was addressed directly to $\mathrm{CM} 2$ rather than everyone in the group chat. However, rather than responding to CF1, CM2 (L37) stated that he felt blessed ('hao xinfu' 好辛福 ) because Dawei had addressed him (L35) as 'Jiujiu（'舅舅maternal uncle), which signalled Dawei had accepted CM2's earlier moves to create a social bond. The sequencing of CF1's interruption, CM2's response and possibly the repeated use of 'Chichu' 吃醋 (jealousy) seems to confuse Dawei, as he wrote in Chinese (L38) 'wo budong' ( 我不懂, I do not understand), followed by two toothy-smile Emojis (L39) and a crying Emoji (L40). Dawei made yet another attempt to identify himself 
(L41) (I am Dawei, 我是大卫), and then started a new exchange - the Paper-Rock-Scissors example presented in Figure 2.

The complex nature of polyadic interaction on WeChat is evident throughout this episode, yet Dawei improvised skilfully with repeated attempts to repair conversational misunderstanding. This suggests high motivation to sustain WeChat conversations and to use Chinese. His aptitude to establish interpersonal bonds through his careful deployment of multiple modes (including language), was consistently striking in our analysis - he privileged the interpersonal and affective dimension of the exchanges, regardless of how little he knew his interlocutors. This finding suggests the need for purpose, motivation and affect in heritage language and literacy learning, as enabled during WeChat interaction with distant family and family friends.

\section{Discussion}

In this paper, we have focussed on the translinguistic, multimodal, polyadic and socialising characteristics of one young, emergent bilingual boy's interaction when using the popular Chinese social media platform WeChat. We have illustrated how the social media platform offered him and his younger brother opportunities to include and learn about their heritage language as part of a broad repertoire of linguistic and semiotic resources they could choose from to interact with distant friends and relations. Our analysis shows how Dawei oriented his WeChat exchanges towards expressing meanings about emotion and social relations. Figures 1, 2 and 4 illustrate how he tended to use primarily image-based modes to express interpersonal meanings, and image-based and written modes to express ideational meanings. All of Dawei's WeChat messages involved him deploying a range of semiotic resources that conveyed emotions, negotiated and affirmed social bonds, and repaired conversational misunderstandings. These patterns of behaviour reflect themes that emerged in the broader dataset. While Daiwei's exchanges arguably did not yet significantly expand his emergent translanguaging in the ideational domain of meaning making, they did provide him with opportunities to communicate in spoken and written Chinese in purposeful and contextualised ways, to strengthen interpersonal relationships with people he knew (e.g. his cousin), and to begin to build relationships with his mother's Chinese speaking friends who he did not know.

Furthermore, engaging in conversations with diverse Chinese-speaking adults in his mother's WeChat networks broadened his access to models of the heritage language. Research has long established that both interaction and access to language models are conducive to language learning (Hoff 2010). In particular, as shown in the data examples, Dawei's WeChat exchanges allowed him to develop sophisticated skills in managing complex multi-partner conversations in written and spoken Chinese, whilst also providing a platform for the creative use of diverse semiotic resources available in social media that he found 'fun'. Reflective of the wider data set, Table A1 clarifies how Dawei chose to use written Chinese in all the WeChat episodes, as part of his rich repertoire of multimodal and translanguaging practices.

In the early stages of our endeavour to understand Dawei's use of the semiotic resources available in WeChat, we found our analysis was constrained by the limitations of wellestablished theories of bi- and multilingualism. We turned to translanguaging theory (Garcia and $\mathrm{Li}$ 2014; $\mathrm{Li} 2018$ ) and found a fit between our data and this practical theory of language that embraces the multilingual and multisensory nature of communication, and is compatible with a social semiotic approach to multimodal analysis. We suggest that our detailed 
descriptive analysis makes an original contribution to translanguaging theory by exemplifying the young boys' emergent translanguaging practices. We choose our phrasing carefully, using 'emergent' to reflect the children's developing competence in written and spoken Chinese (and English) language, developing skills in negotiating complex interactions in social media, as they drew on and expanded their own personal transmodal and translinguistic communicative repertoires. We suggest that Dawei adopted a range of imagebased semiotic resources used typically in social media to support his embryonic mastery of the structural and rhetorical features of Chinese and English. Furthermore, we posit that by bringing together diverse aspects of Dawei's personal history and experiences, his family's networks and practices, WeChat provided a motivating translanguaging space ( $\mathrm{Li} \mathrm{2018)}$ ) for the development of his cognitive and linguistic capacity. By actively observing the WeChat exchanges and occasionally participating, Xiaowei was inducted into online social networking practices. As Rogoff et al. (2003) argue, intent participation such as that exhibited by Xiaowei 'contributes to impressive learning ... and continues in importance throughout life' (p176).

A further significant aspect of the heritage language and literacy practices we observed on WeChat is that they facilitated multiple forms of socialisation and as such offered potential to foster sociocultural identity. Dawei was socialised into his family digital literacy practices through observing his mother typing Pinyin on the iPad and chatting with diverse networks of friends on WeChat. Once he had established WeChat conversations, Dawei actively involved Xiaowei in the interaction, enacting his role as agent (Zukow 1989) for his sibling's socialisation. The WeChat exchanges themselves involved different types of socialisation with familiar and unfamiliar others who shared the same heritage language and culture, and also shared knowledge about English and related cultures. WeChat involved wider socialisation into the peculiarities of digital cultures, where geographically dispersed friends can engage in complex polyadic interactions. In the boys' WeChat exchanges, there was no clear divide between English and Chinese languages, between word-based (written or spoken) or image-based semiotic resources. Rather, the boys enriched their WeChat exchanges by deploying all the semiotic features that were available to them in their complex yet still emergent translanguaging practices.

In summary, through microanalysis of the WeChat data set, we have begun to develop a nuanced understanding of young immigrant children's heritage language and literacy practices on social media, and their skilful multimodal negotiation of intergenerational and interpersonal relationships using diverse semiotic resources. In an age where increasing flows of peoples migrate across territories - whether for economic, social, political, or safety reasons - and where many maintain contact via web-connected digital media - often via social media - we suggest that our multimodal analytic approach, supported by translanguaging theory, could be further developed to understand the rich semiotic repertoires that typify young children's communication across migrant and more fixed populations of all ages, and that shape their emergent transnational and translinguistic identities.

We have approached this study from the perspectives of the children whose practices we observed, and the qualitative nature of our investigation has enabled us to illustrate the complex and performative dynamics of their exchanges on social media. By adopting the concept of translanguaging, we have illustrated how Dawei deployed multiple semiotic resources, including written and spoken Chinese and English, Emojis and stickers, primarily, to express interpersonal and ideational meanings in creative and playful ways as part of his emergent translanguaging practice. We have also shown how Xiaowei observed his older brother's social media activity intently, gradually began to participate actively in the 
exchanges, and was - importantly - occasionally congratulated on his use of Chinese by the distant interlocutors.

\section{Conclusion}

In this paper, we have focussed on young, emergent bilingual children's language and literacy practices on social media in the home environment. Our findings suggest that the boundaries between languages are porous for emergent bilingual children, and that young children are highly resourceful in the ways they draw on a repertoire of linguistic and image-based semiotic resources to enrich their exchanges. We suggest that the development of work in this field could challenge the assumptions of monolingual school-based learning, and have significant implications for the inclusion of heritage languages in the education of emergent multilingual children. For instance, children's creativity as meaning-making agents in the digital environment could be encouraged and used as a springboard to include multilingual practices in early years and early primary classrooms. The study points to the need to develop more nuanced understandings of the role digital and social media play in children's culturally and linguistically diverse lives. It also calls for further exploration of the importance of motivation and affect in heritage language learning, and for research in early childhood and early primary education settings to explore how bilingual and multilingual children deploy translinguistic and transmodal repertoires both at home and in school.

\section{Notes}

1. To ensure alignment between theory and methodology, we followed Eggins and Slade's model, which enabled the coding of conversational moves and showed how Dawei took the lead during exchanges, e.g. using emojis as prolong moves. We subsequently used multimodal analysis to analyse the range of semiotic resources used in the WeChat exchanges. The data annotation indicates where selected episodes occurred within a complete sequence of interaction.

2. Kaomojis, typed on Kana keyboard, is the Japanese emoticon system made up of Japanese characters and punctuation

3. Exchanging red envelopes (with cash inside) is an important Chinese cultural practice. On WeChat, users can give digital red envelops to their conversational partners. Exchanging and 'grabbing' these virtual envelopes in group chats is an established practice.

4. The literal translation for the expression is drinking vinegar, i.e. being sour about something.

\section{Disclosure statement}

No potential conflict of interest was reported by the authors.

\section{References}

Akhter, Parven. 2016. "A young child's intergenerational practices through the use of visual screen-based multimodal communication to acquire Qur'anic literacy." Language and Education 30 (6):500-18. 
Androutsopoulos, Jannis. 2015. "Networked multilingualism: Some language practices on Facebook and their implications." International Journal of Bilingualism 19 (2):185205.

Appadurai, Arjun. 1990. "Disjuncture and difference in the global cultural economy." Theory, culture \& society 7 (2-3):295-310.

Axelrod, Ysaaca, and Mikel W Cole. 2018. "The pumpkins are coming... vienen las calabazas... that sounds funny': Translanguaging practices of young emergent bilinguals." Journal of Early Childhood Literacy 18 (1):129-53.

Bauer, Eurydice Bouchereau, Vivian Presiado, and Soria Colomer. 2017. "Writing through partnership: Fostering translanguaging in children who are emergent bilinguals." Journal of Literacy Research 49 (1):10-37.

Bezemer, Jeff, and Diane Mavers. 2011. "Multimodal transcription as academic practice: A social semiotic perspective." International Journal of Social Research Methodology 14 (3):191-206.

Canagarajah, Suresh. 2011. "Translanguaging in the classroom: Emerging issues for research and pedagogy." Applied linguistics review 2:1-28.

_ 2015. "Clarifying the relationship between translingual practice and L2 writing: Addressing learner identities." Applied linguistics review 6 (4):415-40.

Creese, Angela, and Adrian Blackledge. 2015. "Translanguaging and identity in educational settings." Annual Review of Applied Linguistics 35:20-35.

Dixon, L Quentin, Shuang Wu, and Ahlam Daraghmeh. 2012. "Profiles in bilingualism: Factors influencing kindergartners' language proficiency." Early Childhood Education Journal 40 (1):25-34. 
Dovchin, Sender. 2017. "The role of English in the language practices of Mongolian Facebook users: English meets Mongolian on social media." English Today 33 (2):16-24.

Eggins, Suzanne, and Diana Slade. 2005. Analysing casual conversation. Sheffield: Equinox. Gort, Mileidis. 2006. "Strategic codeswitching, interliteracy, and other phenomena of emergent bilingual writing: Lessons from first grade dual language classrooms." Journal of Early Childhood Literacy 6 (3):323-54.

Gort, Mileidis, and Sabrina Francesca Sembiante. 2015. "Navigating hybridized language learning spaces through translanguaging pedagogy: Dual language preschool teachers' languaging practices in support of emergent bilingual children's performance of academic discourse." International Multilingual Research Journal 9 (1):7-25.

Halliday, M. A. K. 1978. Language as social semiotic : the social interpretation of language and meaning. Baltimore: University Park Press.

Hawkins, Margaret R. 2018. "Transmodalities and transnational encounters: Fostering critical cosmopolitan relations." Applied Linguistics 39 (1):55-77.

Hoff, Erika. 2010. "Context effects on young children's language use: The influence of conversational setting and partner." First Language 30 (3-4):461-72.

Hollingworth, Sumi, Ayo Mansaray, Kim Allen, and Anthea Rose. 2011. "Parents' perspectives on technology and children's learning in the home: social class and the role of the habitus." Journal of Computer Assisted Learning 27 (4):347-60.

Holloway, Donell, Lelia Green, and Sonia Livingstone. 2013. "Zero to eight: Young children and their internet use."

Hu, Jiangbo, Jane Torr, and Peter Whiteman. 2014. "Australian Chinese parents' language attitudes and practices relating to their children's bilingual development prior to school." Journal of Early Childhood Research 12 (2):139-53. 
Jones, Elin Haf Gruffydd, and Enrique Uribe-Jongbloed. 2012. Social media and minority languages: Convergence and the creative industries. Vol. 152: Multilingual Matters. Jordaan, Heila. 2008. "Clinical intervention for bilingual children: An international survey." Folia Phoniatrica et Logopaedica 60 (2):97-105.

Kelly, Clare. 2013. "'Let's do some jumping together': Intergenerational participation in the use of remote technology to co-construct social relations over distance." Journal of Early Childhood Research 13 (1):29-46.

Kenner, Charmian, Mahera Ruby, John Jessel, Eve Gregory, and Tahera Arju. 2008. "Intergenerational learning events around the computer: A site for linguistic and cultural exchange." Language and Education 22 (4):298-319.

Kress, Gunther. 2010. Multimodality: A social semiotic approach to contemporary communication. Lonodn, New York: Routledge.

Kress, Gunther, and Theo Van Leeuwen. 2001. Multimodal discourse: The modes and media of contemporary communication. London, New York: Routledge.

Lankshear, Colin, and Michele Knobel. 2003. New literacies: Changing knowledge and classroom learning: Open University Press.

Li, Wei. 2017. "Translanguaging as a practical theory of language." Applied Linguistics 39 (1):9-30.

Marsh, Jackie, Peter Hannon, Margaret Lewis, and Louise Ritchie. 2017. "Young children’s initiation into family literacy practices in the digital age." Journal of Early Childhood Research 15 (1):47-60. doi: doi:10.1177/1476718X15582095.

Mattheoudakis, Marina, Aspasia Chatzidaki, and Christina Maligkoudi. 2017. "Heritage language classes and bilingual competence: the case of Albanian immigrant children in Greece." International Journal of Bilingual Education and Bilingualism:1-17. 
Page, Ruth. 2018. Narratives Online: Shared Stories in Social Media: Cambridge University Press.

Park, Seong Man, and Mela Sarkar. 2007. "Parents' attitudes toward heritage language maintenance for their children and their efforts to help their children maintain the heritage language: A case study of Korean-Canadian immigrants." Language, Culture and Curriculum 20 (3):223-35.

Pearson, Barbara Zurer. 2007. "Social factors in childhood bilingualism in the United States." Applied psycholinguistics 28 (3):399-410.

Pease-Alvarez, Lucinda. 1993. "Moving In and Out of Bilingualism: Investigating Native Language Maintenance and Shift in Mexican-Descent Children. Research Report: 6." Pennycook, Alastair. 2017. "Translanguaging and semiotic assemblages." International Journal of Multilingualism 14 (3):269-82.

Pontier, Ryan, and Mileidis Gort. 2016. "Coordinated translanguaging pedagogy as distributed cognition: A case study of two dual language bilingual education preschool coteachers' languaging practices during shared book readings." International Multilingual Research Journal 10 (2):89-106.

Reershemius, Gertrud. 2017. "Autochthonous heritage languages and social media: writing and bilingual practices in Low German on Facebook." Journal of Multilingual and Multicultural Development 38 (1):35-49.

Scheele, Anna F, Paul PM Leseman, and Aziza Y Mayo. 2010. "The home language environment of monolingual and bilingual children and their language proficiency." Applied psycholinguistics 31 (1):117-40.

Schwartz, Mila, and Abeer Asli. 2014. "Bilingual teachers' language strategies: The case of an Arabic-Hebrew kindergarten in Israel." Teaching and Teacher education 38:2232. 
Singleton, David, and Larissa Aronin. 2007. "Multiple language learning in the light of the theory of affordances." International Journal of Innovation in Language Learning and Teaching 1 (1):83-96.

Statistica. "Most famous social network sites worldwide as of January 2018, ranked by number of active users (in millions)." Accessed April 2, 2018. https://www.statista.com/statistics/272014/global-social-networks-ranked-by-numberof-users/.

Stewart, Mary Amanda Mandy. 2014. "Social Networking, Workplace, and Entertainment Literacies: The Out - of - School Literate Lives of Newcomer Latina/o Adolescents." Reading Research Quarterly 49 (4):365-9.

Street, Brian V, and Brian B Street. 1984. Literacy in theory and practice. Vol. 9: Cambridge University Press.

Szecsi, Tunde, and Janka Szilagyi. 2012. "Immigrant Hungarian families' perceptions of new media technologies in the transmission of heritage language and culture." Language, Culture and Curriculum 25 (3):265-81.

Velázquez, Isabel. 2017. "Reported literacy, media consumption and social media use as measures of relevance of Spanish as a heritage language." International Journal of Bilingualism 21 (1):21-33.

Verdon, Sarah, Sharynne McLeod, and Adam Winsler. 2014. "Language maintenance and loss in a population study of young Australian children." Early Childhood Research Quarterly 29 (2):168-81.

Wang, Xiqiao. 2017. "Spatial and literacy practices of Chinese international students across a bridge writing classroom and WeChat." Language and Education 31 (6):561-79.

Wong Fillmore, Lily. 2000. "Loss of family languages: Should educators be concerned?" Theory into practice 39 (4):203-10. 
Yu, Qian, Peiying Huang, and Liming Liu. 2017. "From “connected presence” to "panoptic presence": Reframing the parent-child relationship on mobile instant messaging uses in the Chinese translocal context." Mobile Media \& Communication 5 (2):123-38. doi: doi:10.1177/2050157916688348.

Zaman, Bieke, Marije Nouwen, Jeroen Vanattenhoven, Evelien De Ferrerre, and Jan Van Looy. 2016. "A qualitative inquiry into the contextualized parental mediation practices of young children's digital media use at home." Journal of Broadcasting \& Electronic Media 60 (1):1-22.

Zappavigna, Michele. 2018. Searchable Talk: Hashtags and Social Media Metadiscourse. London: Bloomsbury Academic.

Zhou, Baohua, and Shihui Gui. 2017. "WeChat and Distant Family Intergenerational Communication in China: A Study of Online Content Sharing on WeChat." In New Media and Chinese Society, 185-206. Springer.

Zukow, Patricia Goldring. 1989. "Siblings as effective socializing agents: Evidence from Central Mexico." In Sibling interaction across cultures, 79-105. Springer.

Appendix

An Overview of WeChat Episodes

\begin{tabular}{|c|c|c|c|c|c|c|}
\hline \multirow{2}{*}{\multicolumn{2}{|c|}{ Episodes }} & \multicolumn{4}{|c|}{ Participants } & \multirow[t]{2}{*}{ Context and overview } \\
\hline & & Brothers & Intera & $\operatorname{ctant}(s)$ & & \\
\hline \multirow[t]{10}{*}{1.} & Do you & Dawei & CM1 & Adult & Mothers' & A longer exchange initiated by \\
\hline & remember & Xiaowei & & Chinese & friend in & Dawei during the Chinese New Year \\
\hline & me? & & & male & China & with a male friend of his mother \\
\hline & & & & & & who was in China. The friend, $C M 1 *$, \\
\hline & & & & & & had previously talked to Dawei over \\
\hline & & & & & & videophone, which he did not recall. \\
\hline & & & & & & This exchange contains mainly \\
\hline & & & & & & Dawei showing CM1 his Chinese \\
\hline & & & & & & language skills and describing his \\
\hline & & & & & & brother Xiaowei. Xiaowei recorded a \\
\hline
\end{tabular}




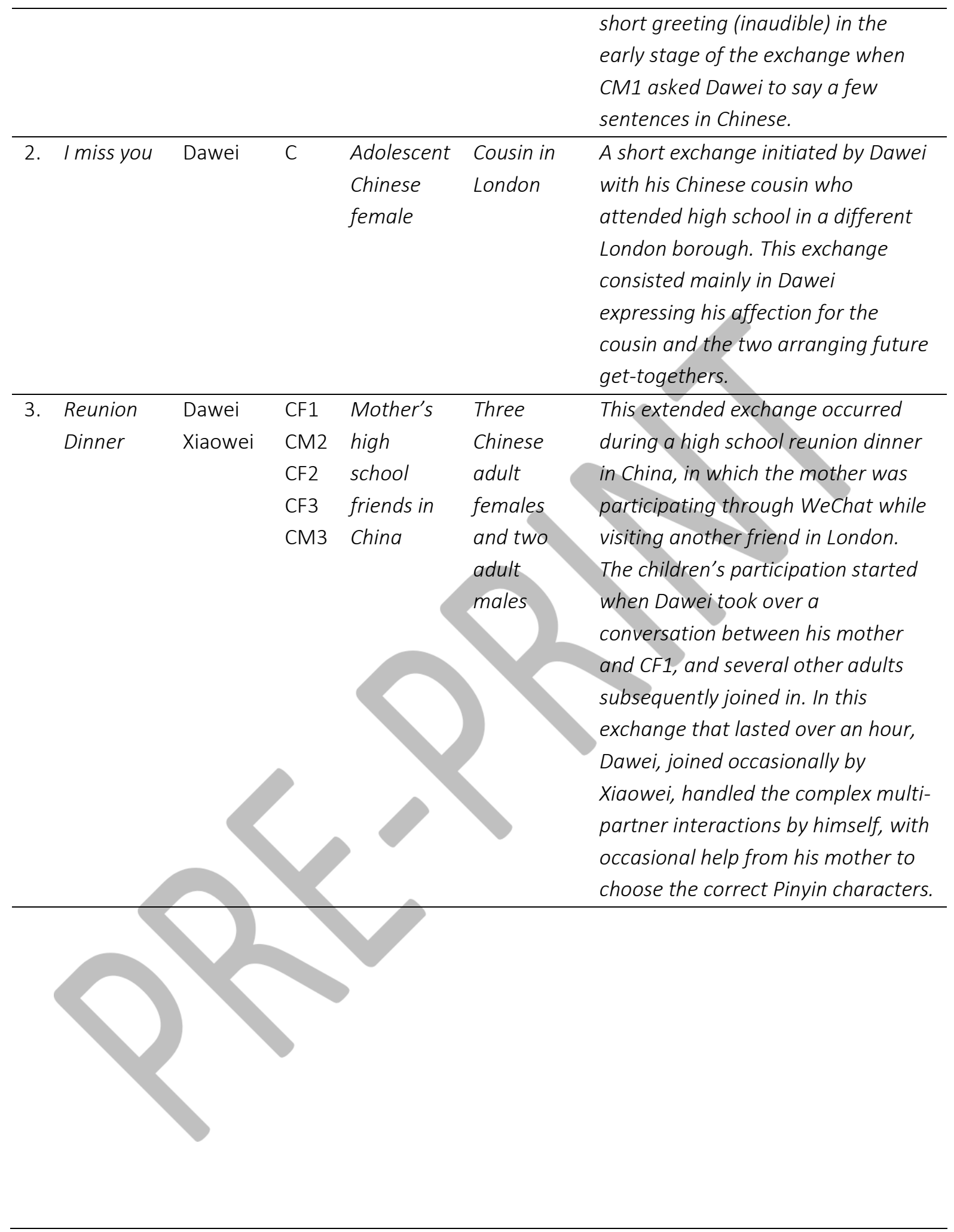

- $C=$ Chinese $M=$ male $F=$ female

*Kaomojis is a type of Japanese emoticon made up of keyboard symbols, e.g. ( ' - ' ). The iPad/iPhone operating system contains a keyboard option for Kaomojis. 
